\title{
Design of a Dynamic Tribometer Applied to Piezoelectric Inertia Drive Motors - In Situ Exploration of Stick-Slip Principle -
}

\author{
Fabien Dubois ${ }^{1,2)^{*}}$, Christian Belly ${ }^{1)}$, Aurélien Saulot ${ }^{2}$ and Yves Berthier ${ }^{2)}$ \\ ${ }^{1)}$ Cedrat Technologies S. A \\ 59 chemin du vieux chêne, Innovallée, 38246 Meylan Cedex, France \\ ${ }^{2)}$ Laboratory of Mechanical Contacts and Structures, Université de Lyon, CNRS, INSA-Lyon, LaMCoS UMR5259 \\ 18-20, rue des Sciences, F-69621 Villeurbanne, France \\ ${ }^{*}$ Corresponding author: fabien.dubois@insa-lyon.fr
}

( Manuscript received 31 August 2015; accepted 19 January 2016; published 30 April 2016 )

(Presented at the International Tribology Conference Tokyo 2015, 16-20 September, 2015 )

\begin{abstract}
In Inertia Drive Motors, generated motion is based on stick-slip principle. Current analytical models are predictive enough to calculate qualitatively their optimal performances, such as maximal step size and speed, with relatively few input parameters. But, they do not take into account the contact life and temporal evolution of parameters as friction factor all along lifetime of IDM. So, analytical models reach their limits when precise predictions are necessary. This investigation aims at understanding wear mechanisms to model temporal evolution of friction. Such an understanding requires the reconstitution of the contact life through the evaluation of $1^{\text {st }}$ and $3^{\text {rd }}$ body flows. To do so, a new IDM-representative tribometer is designed. $1^{\text {st }}$ bodies - coated TA6V and polymer - are not see-through. They are replaced alternatively by an intermediate transparent $1^{\text {st }}$ body to observe the contact dynamically and in-situ. Friction factor, step size and mean speed are also measured. Preliminary results shows that wear profiles from real IDM and tribometer are similar. Direct observations bring out particles of TA6V coating are firstly snatched, then moves in contact and finally trigs others particle detachments.
\end{abstract}

Keywords: stick-slip instabilities, dynamic in situ visualization, contact mechanic, $3^{\text {rd }}$ body behavior

\section{Introduction}

Piezoelectricity is a property of some materials to convert mechanical energy into electrical energy. This phenomenon, called direct effect, has been discovered in 1880 by Pierre and Jacques Curie [1]. As a consequence, piezoelectric elements are used as sensors including strain gauges. Main point of piezoelectricity is its reversibility, called inverse effect, and discovered in 1881 by Gabriel Lippmann [2]. When electrical energy is supplied to piezoelectric elements, they convert it into a micrometric displacement. By neglecting the hysteresis of piezoelectric element [3], it becomes possible to work out a displacement-voltage proportional actuator.

In 1987, Pohl [4] uses the inverse effect to introduce the concept of Stepping Piezoelectric Inertia Drive Motors (IDM) for a scanning tunneling microscope application. A piezoelectric ceramic is attached to an oscillating mass: rubbing $1^{\text {st }}$ body No.1 [5], (Fig. 1(a,b). This $1^{\text {st }}$ body is set in contact, by a spring, with a moving mass: $1^{\text {st }}$ body No.2. Successively, ceramic slow contracts - stick phase: the $1^{\text {st }}$ bodies move together of a few micrometers. Then, it fast expand-slip phase: oscillating mass reaches its original position whereas moving mass remains in its new position. Direction of movement is reversible when expand is slow and contract is fast. Friction is no more synonym of energy loss but becomes a relay in motion transmission. Mastering it aims at controlling movement and energy dissipation into the contact. IDM have then evolved these last thirty years [6-8] to branch out into many industrial sectors such as optronic [9] and medical applications [10]. These motors are useful in embedded applications where celerity, force and volume are low (respectively a few centimeters per second, a few newtons and lower than 15 cubic centimeters) and when micrometric resolution positioning is necessary [11].

Depending on application, specific constraints, such as no wear generation or biocompatibility, must be respected. Here, biocompatibility $[12,13]$ and wear are bound by the tribological circuit [14]. System configuration was decided to set the $1^{\text {st }}$ body that wears the most in macroscopic moves to avoid excessive wear 
a

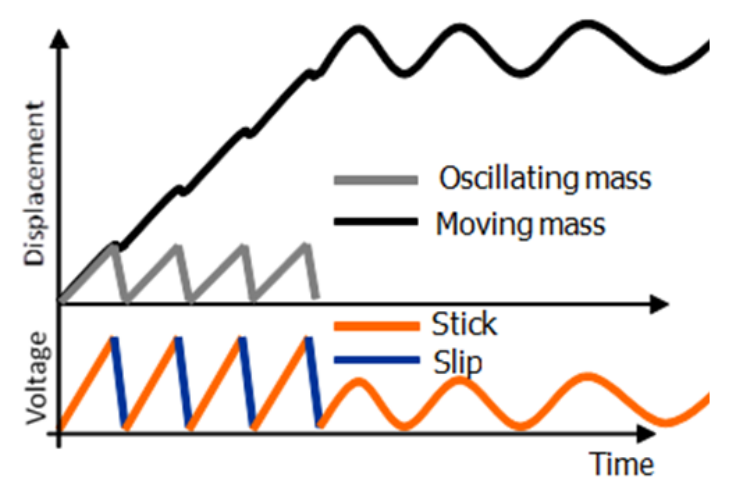

b

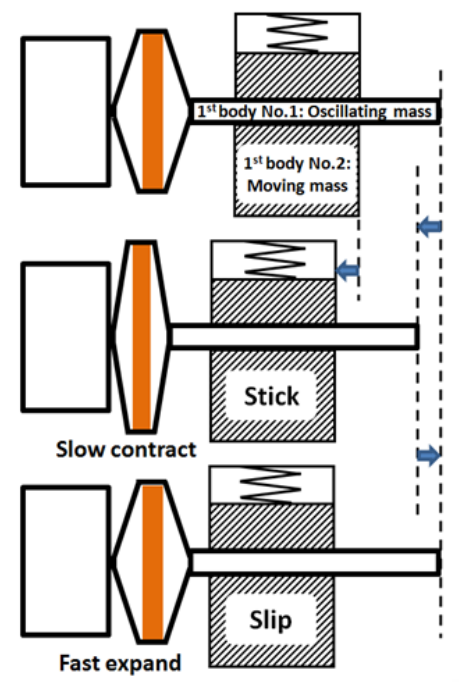

C

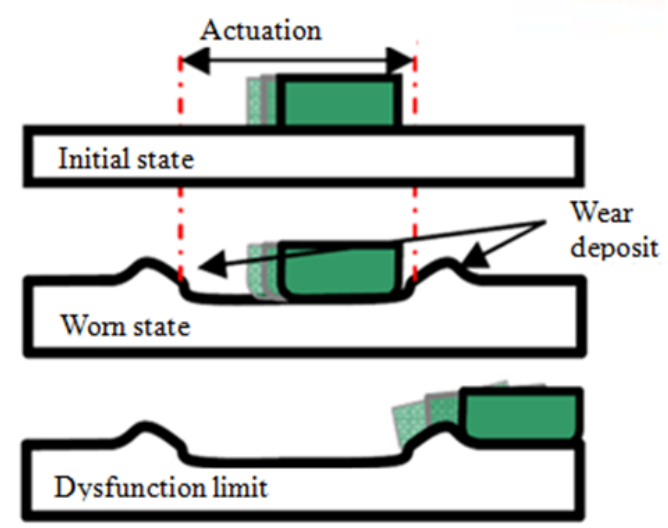

Fig. 1 IDM principle: (a) Proportionality between voltage order and piezoelectric microscopic displacement. (b) Microscopic to macroscopic displacement by stick-slip steps sequence. (c) Wear mechanism when polymer makes macroscopic moves along TA6V stroke

particle deposit leading to dysfunction (Fig. 1(c)).

This investigation focuses on the understanding of this wear mechanism through the evaluation of $1^{\text {st }}$ and $3^{\text {rd }}$ body flows to get the overall understanding of the tribological system. It is based on Linear Stepping Piezo Actuator (LSPA, Fig. 2) from Cedrat Technologies company [15].

An analytical LuGre model of macroscopic friction

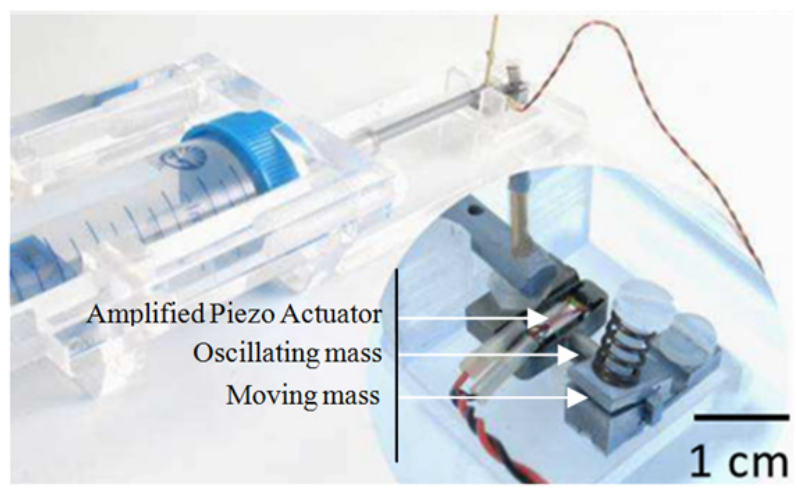

Fig. 2 Cedrat Technologies IDM. LSPA30 $\mu$ XS, oscillating shaft, $1^{\text {st }}$ body No. 1 , is in polymer and moving clamp, $1^{\text {st }}$ body No. 2 , is in coated TA6V

has been introduced in [16]. Although qualitative prediction is good to calculate optimal performances, a time representative limitation has been identified. Friction coefficient is used as an input static parameter without taking into account wear of the interfaces. A new tribometer is introduced to raise that limit.

Dynamic and in-situ observations give an overview of what happens in contact, and so reconstitute the associated tribological circuit, by replacing successively $1^{\text {st }}$ bodies with transparent ones. The comparison between measurements and observations led to a better understanding of the $3^{\text {rd }}$ body behavior in IDM. This investigation is completed by a discussion about the use of an intermediate transparent $1^{\text {st }}$ body and its impact on the analysis.

\section{Material and methods}

\subsection{Studied IDM: LSPA30 $\mu$ XS}

Linear Stepping Piezoelectric Actuators (LSPA) are composed of an Amplified Piezoelectric Actuator $\left(\mathrm{APA}^{\circledR}\right)$ [15] whose oscillating mass, $1^{\text {st }}$ body No.1, is a shaft, and whose moving mass, $1^{\text {st }}$ body No. 2 , is a clamp. Coated TA6V-polymer rubbing couple is used in LSPA-30 $\mu$ XS design (Fig. 2). Geometry of shaft is rectangular. Material is non disclosed polymer. Clamp interface is four convex half cylinders $3 \mathrm{~mm}$ radius. Preload is provided by a spring whose stiffness is 2.5 $\mathrm{kN} / \mathrm{m}$. Mean Hertz contact pressure is 4.2 MPa.

\subsection{Contact life: A new dynamic in-situ IDM tribometer}

\subsubsection{Tribometer design constraints}

Through the $3^{\text {rd }}$ body concept, first constraint is to link friction factor evolution to direct observations. Purpose is to understand the life contact with a mechanical approach and being able to attribute accommodation sites and modes [14]. Current $1^{\text {st }}$ bodies are not see-through. First, friction factor with coated TA6V against polymer is measured. Then, $1^{\text {st }}$ bodies are alternatively replaced by transparent one to see through. By finally comparing direct in-situ observations, 
microscopy analyses and measurements, it becomes possible to get sought tribological circuit. Design of the tribometer must provide an oscillating part with a hole and space to glue - like in a real IDM - rubbing samples.

Second challenge was to find an optimal size of the tribometer, allowing performing all the measurements at the same time while remaining at IDM scale. The main point was to maintain a representative $3^{\text {rd }}$ body life inside the contact. If the tribometer is too large, global speed would decrease and local $3^{\text {rd }}$ body behavior would change. If the tribometer is too small, there is not space enough to set all the sensors. Therefore, mass and volume of the bench has been optimized to keep same orders of magnitude than LSPA: actuation force $(2 \mathrm{~N})$, speed $(10 \mathrm{~mm} / \mathrm{s})$, contact pressure $(1-10 \mathrm{MPa})$ and actuation frequency $(1 \mathrm{kHz})$. Contact has also been simplified. Instead of four into LSPA30 $\mu$ XS configuration, clamp and shaft has been replaced by a pin on pad contact (Fig. 3) whose geometry is that of LSPA30 $\mu$ XS - cylinder/flat contact. Spring preload has been kept with a $2.5 \mathrm{kN} / \mathrm{m}$ stiffness order of magnitude.

Finally, guiding must be considered to ensure linear movement without dissipating too much energy. New Way air guiding has been chosen for their normal stiffness - $4 \mathrm{~N} / \mu \mathrm{m}$ - and their friction factor is negligible because there is no contact with moving mass (Fig. 3). Three $12 \mathrm{~mm} \times 24 \mathrm{~mm}$ flat rectangular air guiding (Fig. $5(\mathrm{~g}))$ are integrated in dovetail to both ensure guiding, lift and limiting the number of air input.

\subsubsection{Tribometer instrumentation}

All measurements and associated sensors are listed in Table 1 and illustrated (Fig. 4(a)). National Instrument USB-6259 BNC electronic board (Fig. 4(b)) associated with Labview (Fig. 4(c)) is used to generate actuation signal and acquire measurement data.

Piezoelectric ceramic can be compared to electrical capacitance. Current consumption depends on voltage

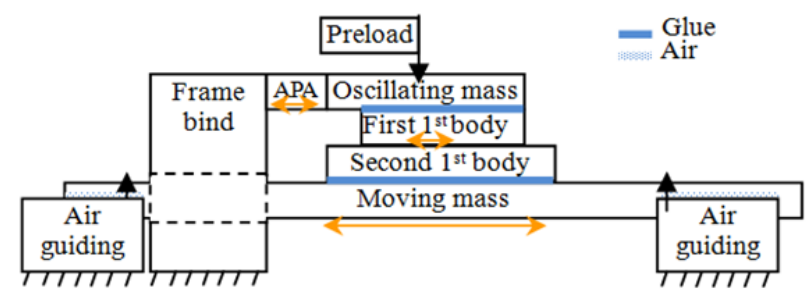

Fig. 3 Principle scheme of tribometer. $1^{\text {st }}$ bodies are bound to the moving and oscillating mass by the same glue as that real IDM. Guiding is provided by air to minimize parasitic friction and only studying friction between $1^{\text {st }}$ bodies. Preload is provided by a spring like in real IDM. Double arrows: APA generates small amplitude dissymmetric oscillations. $1^{\text {st }}$ body No.1 relays and converts these small oscillations in macroscopic movement through stick-slip principle. and frequency of actuation signal. If current limitation of power supply is too low $(<471 \mathrm{~mA}$ for a $2 \mathrm{kHz}$ and $150 \mathrm{~V}$ actuation signal here), mechanical performances are decreased. Linear amplifier (Cedrat Technologies LA75C) (Fig. 4(d)) is used with 2.4 ampere current limitation. Voltage and current (Fig. 4(e,f)) are measured to control power supply.

Since actuation frequency is $1 \mathrm{kHz}$ to keep same order of magnitude with LSPA, common camera are not relevant for direct observations, high speed camera is used instead. A Vision Research V710 high-speed camera (Fig. 4(g)) associated with PCC2.6 software (Fig. 4(h)) is used with $5 \mathrm{kHz}$ frame per second,

Table 1 List of integrated measurement in test bench

\begin{tabular}{l|l}
\hline \multicolumn{1}{c|}{ Measures } & \multicolumn{1}{c}{ Sensors } \\
\hline Friction factor: & \\
- Normal force & Strain gauge \\
- Tangential force & Optical sensors + processing \\
\hline $\begin{array}{l}\text { Direct observations: } \\
\text { - In-situ }\end{array}$ & $\begin{array}{l}\text { Camera through lenses and } \\
\text { - Migh speed camera }\end{array}$ \\
\hline $\begin{array}{l}\text { Linear momentum: } \\
\text { - Before friction } \\
\text { - After friction }\end{array}$ & $\begin{array}{l}\text { Differential laser-vibrometer } \\
\text { sensor }\end{array}$ \\
\hline $\begin{array}{l}\text { Initial \& Final } \\
\text { conditions: }\end{array}$ & Microscope/SEM-EDX \\
\hline
\end{tabular}

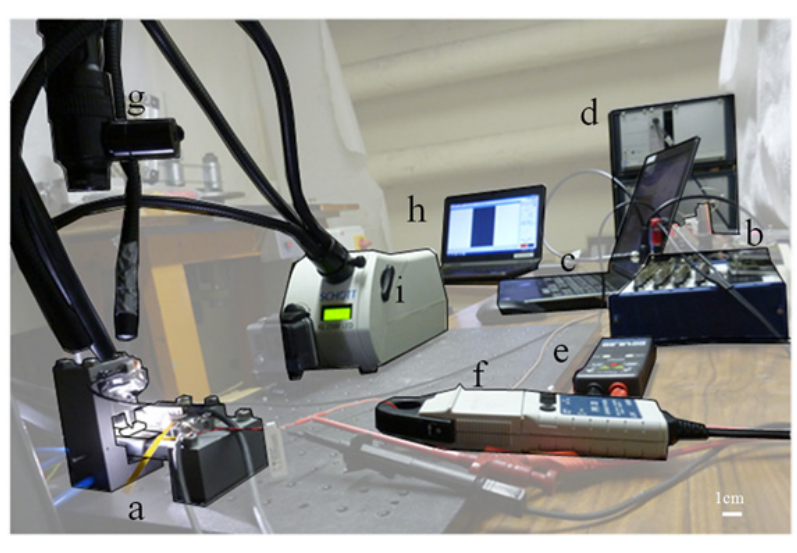

Fig. 4 Global SPA-tribometer setup. (a) Tribometer setup. (b) Schott KL 2500 LED. (c) Current probe to get input electrical energy. (d) Voltage probe to get input electrical energy. (e) National Instrument NI USB-6259 BNC acquisition and generation card. (f) LA75C Cedrat Technologies power supply and SG75 strain gauge controller. (g) Labview computer manage signals and post-treatment. (h) V710 Vision Research high speed camera and Navitar6000 optical zoom. (i) High speed camera computer 
associated with a Navitar 6000 optical zoom to focus on the contact and a Schott KL2500 Led (Fig. 4(i)) to provide enough light without harming contact. Observation window is $8 \mathrm{~mm} \times 5 \mathrm{~mm}$ with $360 \times 480$ pixels. Each pixel measures $15 \mu \mathrm{m}$ by side. Step size is $75 \mu \mathrm{m}$ so there are 5 pixels by step.

To complete analysis of the friction interface (Fig. 5(a)), a test body has been designed [17] to measure normal force in a $[3 \mathrm{~N} ; 9 \mathrm{~N}$ ] range with a HBM strain gauge - 2 half-bridges (one on each side of the test body) referenced 1-DA53K3.2/350_E (Fig. 5(b)). Normal stiffness is $41.2 \mathrm{kN} / \mathrm{m}$. Tangential forces and relative displacements of $1^{\text {st }}$ bodies are measured with Numerik Jena optical sensors (Fig. 5(e,f)). Characteristics are a 3 millimeters stroke, $0.1 \mu \mathrm{m}$ resolution and $30 \mathrm{kHz}$ sampling.

Mechanical representativity of the contact is finally controlled by the spring preload, like in real IDM with a global normal stiffness of $2.3 \mathrm{kN} / \mathrm{m}$ in the normal direction which is closed to the IDM stiffness: 2.5 $\mathrm{kN} / \mathrm{m}$.

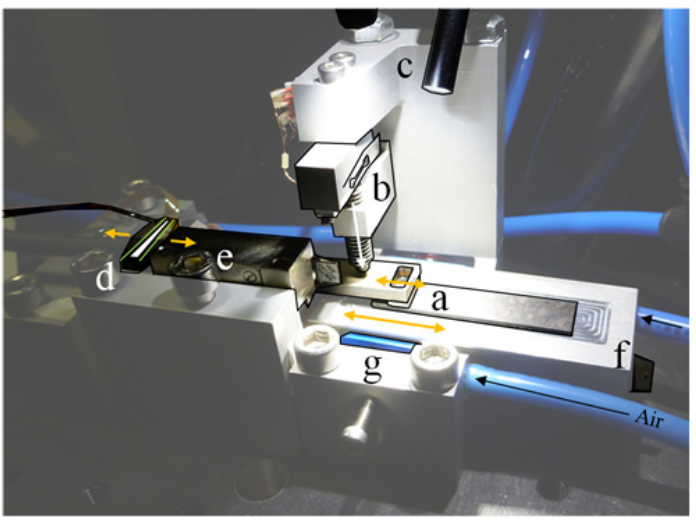

Fig. 5 Tribometer setup. (a) $1^{\text {st }}$ bodies contact. Current geometry is semi-circular pin on pad. (b) Preload function (write arrow) is provided by a spring contained in a screw. Applied force is proportional to screw position and measured by a strain gauge mounted on specific deformable test body. Applied Normal force range is [3 N; $10 \mathrm{~N}$ ]. (c) Extra light is provided by $\mathrm{Schott}^{\circledR} \mathrm{KL}$ 2500 LED to make high speed camera acquisition possible without harming contact. (d) Piezoelectric actuator linked to oscillating mass. Strain gauge is mounted on actuator to get actuation force. (e) Numerik Jena optical sensor to get displacement, speed and acceleration of oscillating mass. Sampling is $30 \mathrm{kHz}$, resolution is $0,1 \mu \mathrm{m}$. (f) Numerik Jena optical sensor to get displacement, speed and acceleration of moving mass. Sampling is $30 \mathrm{kHz}$, resolution is $0,05 \mu \mathrm{m}$. (Only scale tape is seen into the moving mass). (g) Guiding and lift are provided by New way air bearings

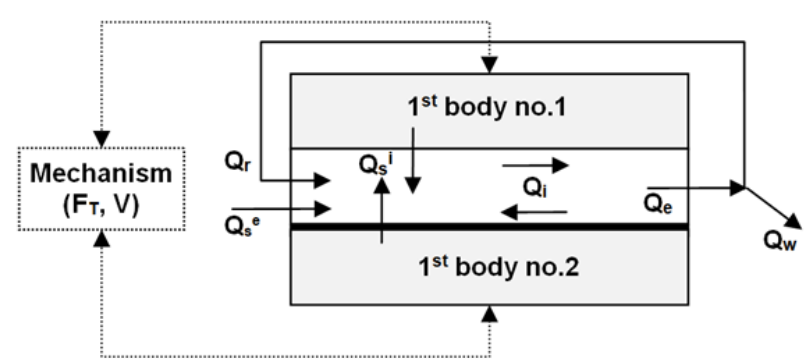

Fig. 6 Tribological circuit principle scheme. $3^{\text {rd }}$ body particles move with velocity gradients. They are described in terms of flows. The determination of $3^{\text {rd }}$ body origin - $Q_{\mathrm{s}}{ }^{\mathrm{i}}$ and $Q_{\mathrm{s}}{ }^{\mathrm{e}}$-, and phenomena implied in the activation of different flows - $Q_{\mathrm{e}}, Q_{\mathrm{r}}, Q_{\mathrm{w}}$ and $Q_{\mathrm{i}}$ - are essential to understand lifetime of IDM

\subsection{3rd body approach}

Godet and Berthier proposed a phenomenological concept from experimental observations called the tribological triplet [5]. When two $1^{\text {st }}$ bodies are in contact and in relative motion, an interfacial layer creates - called $3^{\text {rd }}$ body - which is subjected to speed gradient. Such $3^{\text {rd }}$ body flows inside the contact [18]. Wear is approached in term of $3^{\text {rd }}$ body flow. The different flows are illustrated in Fig. 6. $Q_{\mathrm{s}}{ }^{\mathrm{i}}$ is internal source of $3^{\text {rd }}$ body, got by particle detachment from $1^{\text {st }}$ bodies. $Q_{\mathrm{s}}{ }^{\mathrm{e}}$ is external source got by environment particle detachment. $Q_{\mathrm{i}}$ is internal flow of particle inside the contact. $Q_{\mathrm{e}}$ is ejection flow divided in $Q_{\mathrm{r}}$ and $Q_{\mathrm{w}} . Q_{\mathrm{r}}$ is recirculation flow when particle left the contact and are introduced again then. $Q_{\mathrm{w}}$ is wear flow for particles leaving definitively the contact.

$3^{\text {rd }}$ body causes the activation of the different flow rates in the tribological system. Highlighting it is essential to control lifetime of IDM.

\section{Measurements: tribometer high speed observations}

A Labview program has been used to generate thousand back and forth of three steps. Each step lasts 2 $\mathrm{ms}-1 \mathrm{~ms}$ for actuation signal and $1 \mathrm{~ms}$ of dead time to damp free oscillations - so total acquisition lasts 12 seconds. High speed camera is set to 5000 frame per second (fps). It is compromised between getting a lot of frames per actuation period and light power. More fps means less aperture time ( $0.1 \mathrm{~ms}$ currently) so less visibility to observe the contact.

Tests are divided in three phases, one per material couple: Coated TA6V/Polymer, Glass/Polymer and Glass/Coated TA6V. In each phase, five sessions of actuations are performed which leads to five thousands back and forth. Observations on both glass/polymer and glass/coated TA6V are used to reconstitute polymer/coated TA6V wear mechanisms. 


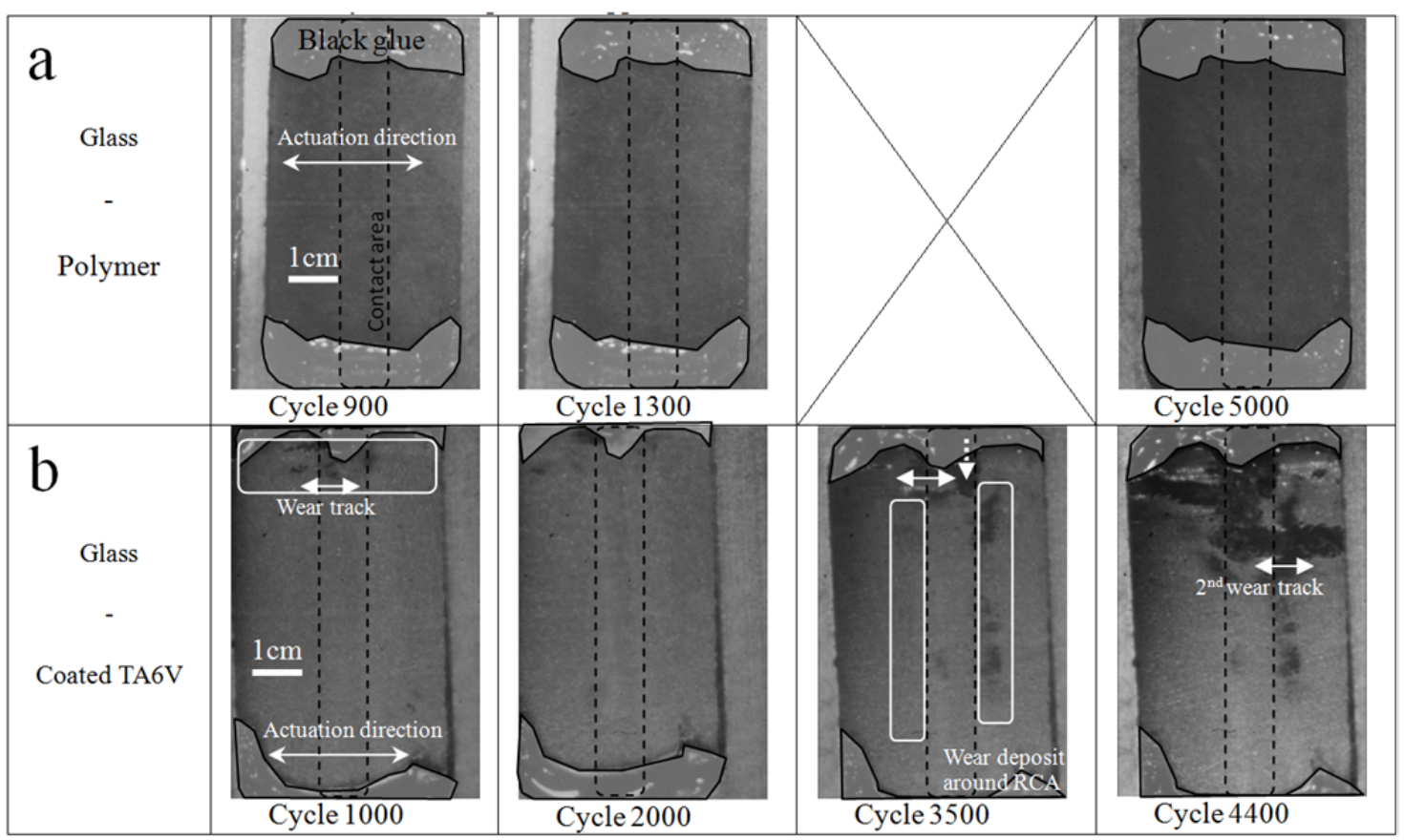

Fig. 7 High speed camera observations. Glass on polymer actuation does not make appear any flow. Glass on coated TA6V trigs $Q_{\mathrm{s}}{ }_{\mathrm{i}}$ after 1000 cycles. $Q_{\mathrm{i}}$ is activated after 2000 cycles and deposit appears around real contact area. After 3500 cycles, $Q_{e}, Q_{s}{ }^{\mathrm{e}}$ and $Q_{\mathrm{w}}$ are activated. After 4400 cycles, $3^{\text {rd }}$ body particles generate a second track

\subsection{Glass pin / polymer pad}

All along the five thousands back and forth, no flow is visibly activated (Fig. 7(a)).

\subsection{Glass pin/TA6V pad}

From the first thousand cycles, dark particles appear (Fig. 7(b)). Then, these particles move in actuation direction until the end of the test. Black spot appears on glass pin and a bright track full of particles remains on the pad after the contact. Around 3500 cycles moves perpendicularly to the actuation direction and trigs occurrence of a new wear track in the actuation direction.

Around two thousand cycles, edges of real contact appears. Particle deposit along these edges increases permanently until the end of the test.

\section{Results}

4.1. Tribometer representativity

Macroscopic representativity of the tribometer is

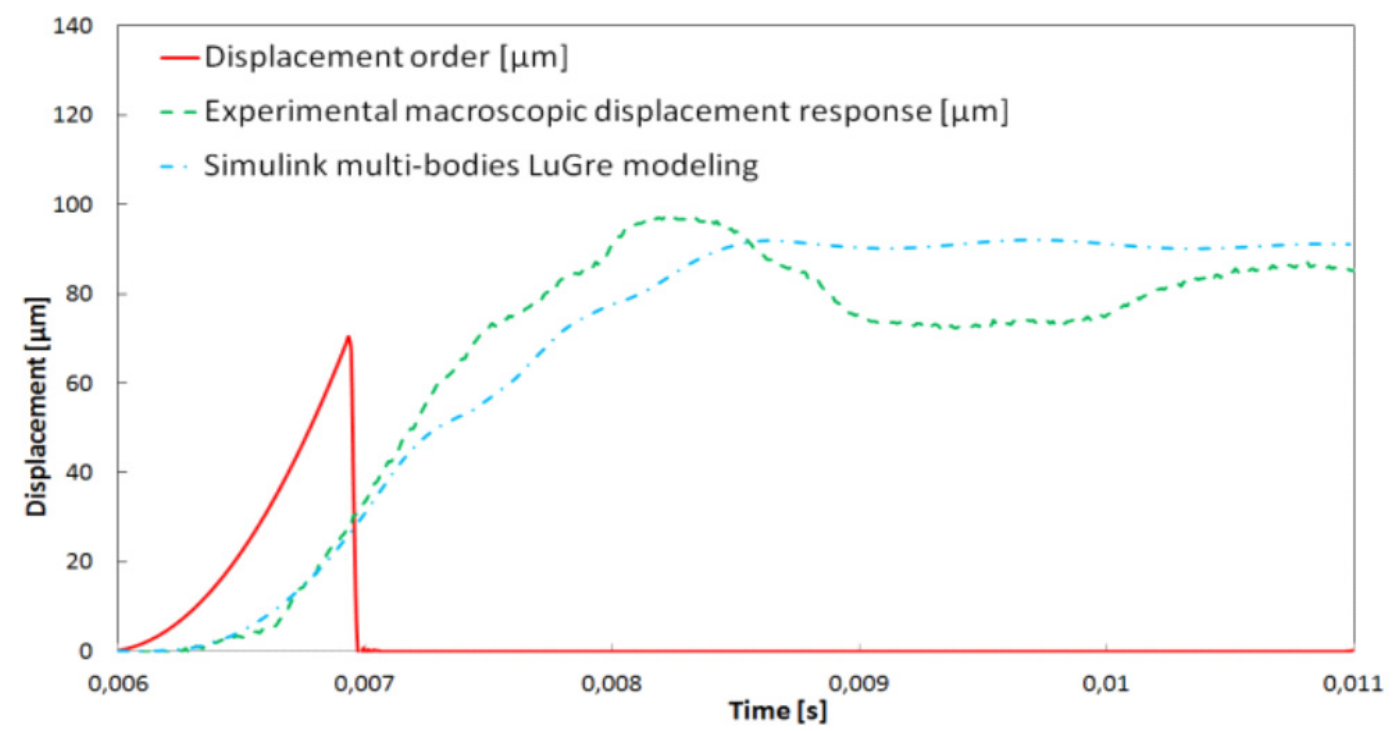

Fig. 8 Comparison between experimental measurements and Simulink analytical modeling before tests.

Experimental step size is $83 \mu \mathrm{m}$ and modeled step size is $90 \mu \mathrm{m}$ 
analyzed by comparing macroscopic displacement of moving mass with what is expected in modeling of real IDM. By design, mass of oscillating part and moving part are respectively $12 \mathrm{gr}$ and 34 gr. Friction factor has been measured with polymer against coated TA6V before tests: $0.41+/-0.04$. Preload has been set to $5 \mathrm{~N}$. Mean Hertz contact pressure is $6.9 \mathrm{MPa}$ which is closed to LSPA reality. These parameters are typically used as input data into Simulink modeling to describe real IDM behavior. Steps are measured and plotted in (Fig. 8). A

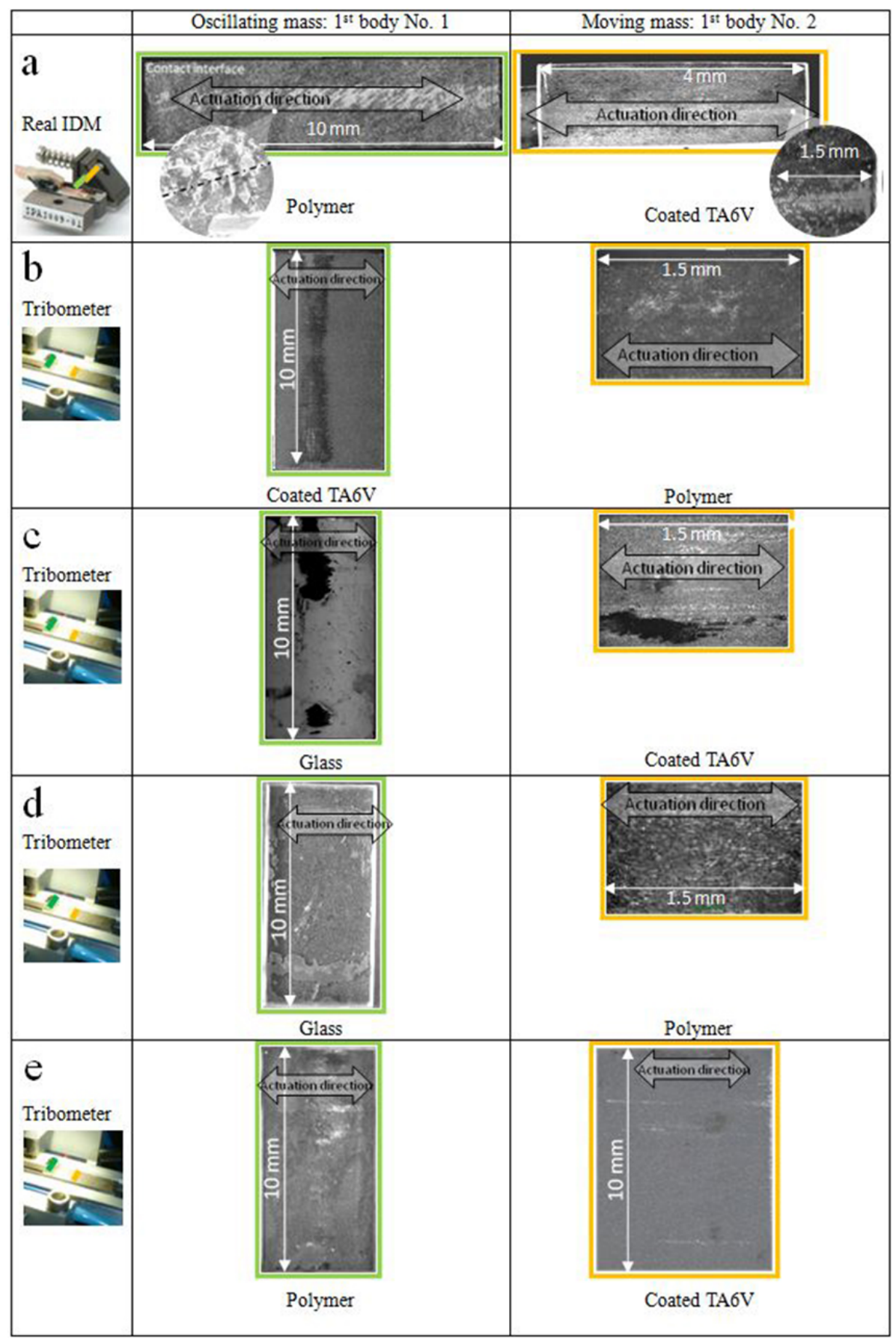

Fig. 9 Wear profile comparison after 5000 cycles. (a) Real IDM. On the polymer side, black matrix of the polymer is brighter in wear track. Zoom shows many little bright fibers in track and a few bigger fibers outside the track. On the coated TA6V side, $1^{\text {st }}$ body appears under coating in wear track and a deposit of dark particles surrounds it. (b) Tribometer - coated TA6V/polymer. Coated TA6V and polymer wear profiles are similar as in real IDM. (c) Tribometer - glass/coated TA6V. Particles of coating are trapped in pin. Wear track in actuation direction appears on these particles only. (d) Tribometer - glass/polymer. Neither glass nor polymer is worn. (e) Tribometer - polymer/coated TA6V. As in (b), coated TA6V and polymer wear profiles are similar as in real IDM 
$400 \mathrm{~Hz}$-mode appears during the free oscillation phase. This low frequency mode does not appear in the analytical modeling due to the tribometer conception different from the real IDM conception on which the model is based. Investigations are in progress to determine what the differences are responsible for this mode occurrence. Error on displacement step size is $8.4 \%$ which is relevant enough to confirm macroscopic representativity of the tribometer.

Microscopic representativity of the tribometer is analyzed at the scale of the contact. After tests, $1^{\text {st }}$ bodies are observed with optical microscopy, detailed below (Fig. 9). In real IDM (Fig. 9(a)) like in tribometer (Fig. 9(b,e)), when polymer rubs against coated TA6V, wear profiles are similar. Coating is snatched from the TA6V substrate and remains on polymer. Tribometer is also representative of a real IDM at the scale of contact.

\subsection{Wear mechanism}

Through glass pins, direct in situ observations give information about what happens in contact with similar solicitations of real IDM such as typical stick-slip actuation. When glass pin is actuated against polymer pad, none flow is visibly activated at this scale of observation (Fig. 7(a)) and no macroscopic wear appears (Fig. 9(d)). In case of glass pin against coated TA6V pad, for the first thousand cycles (Fig. 7(b)), black particles detachment is supposed to be that of coating because glass is transparent. These particles are trapped in glass and wear track appears on it in actuation direction (Fig. 9(c)). $Q_{\mathrm{s}}{ }^{\mathrm{i}}$ from coating is activated. Then $Q_{\mathrm{i}}$ is activated with $3^{\text {rd }}$ body particles newly generated. Particles stick both interfaces and moves with contact in actuation direction. Wear track has same aspect as TA6V - that of pad without coating so it is presumed to be $1^{\text {st }}$ body substrate emergence. First tests have shown flow rates are mainly activated from coated TA6V. Tests have been reproduced two times between glass pin and coated TA6V pad to confirm preliminary observations (Fig. 10). Same wear mechanism is again observed. $Q_{\mathrm{s}}{ }^{\mathrm{i}}$ is first activated from coated TA6V and then move with actuation direction. Pulverulent particles are generated and locate lift into the contact. Then, these particles snatch other particles always in actuation direction until a threshold is reached. After reaching this threshold, particles migrate perpendicularly to the actuation direction toward the center of the visualization window. Then, they go on moving with the actuation direction. It has also been possible to see particles becoming cohesive and sheared under friction action giving information about speed accommodation mechanism.

Preliminary post-mortem observations, with optical microscopy, give information about aspect of interfaces and $3^{\text {rd }}$ body behavior. First, on the glass pin side, which rubbed against coated TA6V pad, main particle volume is in the high part of the visualization window (Fig. 9(c)). Particles are both composed of coating - dark particles - and TA6V oxide substrate - bright particles. All along the contact area, cohesive particles alternates with pulverulent one which is consistent with the thresholds seen in in-situ dynamic observations. On the coated TA6V pad side, substrate - bright areas - appears under pulverulent and cohesive coating particles. Some TA6V particles appear also above coating particles. So $3^{\text {rd }}$ body is not only composed of coating but also with

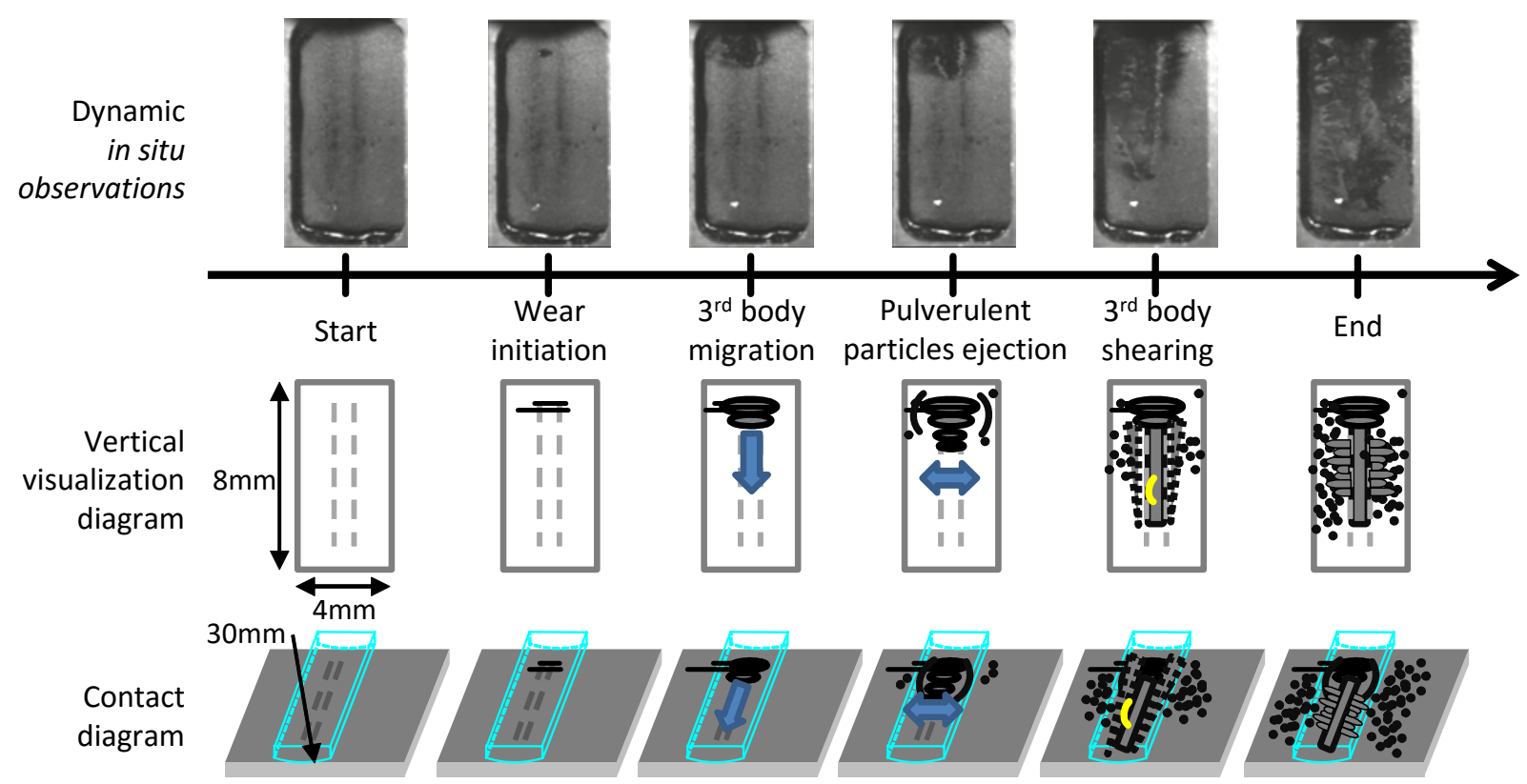

Fig. 10 Synthesis of direct in situ visualization glass pin on coated TA6V pad confirmation test. Like in Fig. 7, $Q_{\mathrm{s}}{ }^{\mathrm{B}}$ is first activated. Then generated $3^{\text {rd }}$ body migrates $\left(Q_{i}\right)$ toward the center of the visualization window. Finally, particles go on moving with the actuation direction again and cluster of cohesive particles are sheared in the actuation direction 
substrate. Second, In case of glass pin against polymer pad, no wear is visible at this scale (Fig. 9(d)). So when glass is used as a pin, polymer is definitely not a source of flow activation but coating from coated TA6V is. Third, in case of coated TA6V pin against polymer pad (Fig 9(b)), after five thousands cycles, actuation track on pin is as bright as coated TA6V pin without coating but there is no deposit outside ( $Q_{\mathrm{w}}$ is not activated) so particles remained in contact. Finally, in case of polymer pin against coated TA6V pad (Fig. 9(e)), cohesive particle clusters are visible on the pin. Like with glass pin against coated TA6V pad, the main volume of particles on the polymer pin is in the high part of the visualization window and decrease toward the center. Some substrate bright particles are visible in the center of the windows. On the coated TA6V pad side, some bright tracks appear in the same direction as actuation but aspect is less worn than polymer pin. This gives information on the dynamic because substrate is under coating so must appear after in the wear mechanism. So $3^{\text {rd }}$ body flow initiates at the edge and then move on toward the center of the contact in both intermediate and real rubbing interfaces cases.

From these preliminary observations, wear mechanism into coated TA6V pin against polymer is deduced. Coating is quickly snatched from TA6V $1^{\text {st }}$ body and moves in the actuation direction. These particles are trapped into the fibers of polymer. Lift and speed accommodation are no more located in screen interfaces but in $3^{\text {rd }}$ body. By actuation, some of the trapped particles agglomerate into cohesive cluster. Then, they move with contact and snatch other particles. Some others, more pulverulent, remains in the wear track and are reintroduced when pin go on again. Polymer has a role of $3^{\text {rd }}$ body particles trap and limits $Q_{\mathrm{w}}$.

\section{Conclusion}

Using an intermediate transparent sample made possible a direct in-situ analysis but this point must be discussed. Chosen material of intermediate body is glass because silicon oxide is in the chemical composition of the polymer and coating. Optical properties made possible direct observations. Parts were geometrically designed as a function of material properties to respect similar contact pressure. Roughness is as low as possible $\left(R_{\mathrm{a}}=5 \mathrm{~nm}\right)$ to not disrupt optical signal. Resulting direct observations, consistent with real IDM wear profiles, have validated the use of these intermediates parts. Thus, tribometer tribological realism is validated.

Preliminary results are encouraging. As expected, macroscopic step size and wear profiles were similar between analytical modeling, real IDM behavior and tribometer behavior.

Observations show how wear mechanism is activated in case of coated TA6V against polymer. In first case, coating particles are quickly detached from coated TA6V. Then, these particles snatch other ones, and are trapped into fibers on the polymer side. Now the representativity of the tribometer is demonstrated, mechanism that trigs the first detachment must be understood. It means including the accommodation modes [18] in the investigation. These observations will be confirmed by a wider test campaign. Complete wear mechanism will be available once rubbing interfaces have been analyzed with chemical analysis trough Scanning Electron Microscopy associated with energy dispersive X-ray spectroscopy. Furthermore, FEM model of the tribometer will be worked out to give access to the stress distribution near and far away from the contact and to link it with previous observations.

\section{References}

[1] Curie, J. and Curie, P., "Development, by Pressure, of Polar Electricity in Hemiedric Crystals with Inclined Faces," Journal de Physique Théorique et Appliquée, 91, 1880, 294-295 (in French).

[2] Lippmann, G., "Principle of Electricity Conservation, or Second Principle of Electrical Phenomena Theory," Journal de Physique Théorique et Appliquée, 10, 1, 1881, 381-394 (in French).

[3] Breguet, J. M., "Actuators "Stick and Slip" for Micro-Manipulators," PhD thesis, EPFL, 1998 (in French).

[4] Pohl, D. W., "Sawtooth Nanometer Slider: A Versatile Low Voltage Piezoelectric Translation Device," Surface Science, 181, 1-2, 1987, 174-175.

[5] Godet, M., "The Third-Body Approach: A Mechanical View of Wear," Wear, 100, 1-3, 1984, 437-452.

[6] Morita, T., "Miniature Piezoelectric Motors," Sensors and Actuators A: Physical, 103, 3, 2003, 291-300.

[7] Belly, C., Bagot, M. and Claeyssen, F., "High Resolution Actuators for Severe Environments," Proc. Precision Assembly Technologies and Systems, Chamonix, France, 371, 2012, 89-96.

[8] Belly, C., Buttery, M. and Claeyssen, F., "Thermal Vacuum Behaviour of a Stepping Piezo Actuator," Proc. European Space Mechanisms and Tribology Symposium, Constance, Germany, 653, 23, 2011, 195-199.

[9] Belly, C., Porchez, T., Dubois, F. and Barillot, F., "Long Stroke/High Resolution Tip Tilt Mechanism,” In Proc. Actuator, Bremen, Germany, 2014.

[10] Khalaji, I., Hadavand, M., Asadian, A., Patel, R. V. and Naish, M. D., "Analysis of Needle-Tissue Friction During Vibration-Assisted Needle Insertion," In Intelligent Robots and Systems (IROS), Tokyo, Japan, 2013, 4099-4104. 
[11] Belly, C. and Charon, W., "Benefits of Amplification in an Inertial Stepping Motor," Mechatronics, 22, 2, 2012, 177-183.

[12] Chinzei, K., Kikinis, R. and Jolesz, F. A., "MR Compatibility of Mechatronic Devices: Design Criteria," In Medical Image Computing and Computer-Assisted Intervention - MICCAI'99, 1679, 1999, 1020-1030.

[13] Von Oldenburg, G. and Speitling, A. D., "Osteosynthetic Device of Steel, Cobalt and/or Titanium Alloy," European Patent 1228775 A3, 2002.

[14] Colas, G., Saulot, A., Godeau, C., Michel, Y. and Berthier, Y., "Decrypting Third Body Flows to Solve Dry Lubrication Issue - MoS2 Case Study under Ultrahigh Vacuum," Wear, 305, 1-2, 2013, 192-204.

[15] Claeyssen, F., Ducamp, A., Barillot, F., Le Letty, R., Porchez, T., Sosnicki, O. and Belly, C., "Stepping Piezoelectric Actuators Based on APAs," In Proc. Actuator, Bremen, Germany, 2008.

[16] Belly, C., "Inertial Piezoelectric Motors: Designs, Achievements, and Test Applications," $\mathrm{PhD}$ thesis, Université de Technologie de Belfort-Montbeliard, 2011 (in French).

[17] Henein, S., "Flexible Guiding Design," Presses Polytechniques et Universitaires Romandes, 2001 (in French).

[18] Berthier, Y., "Maurice Godet's Third Body," Tribology series, 31, 1996, 21-30. 